\title{
Dynamical Analysis of Dissipative Collisions between Ar and Ag nuclei in the Fermi Energy Domain
}

\author{
F. Haddad ${ }^{1,2}$, B. Borderie ${ }^{3}$, V. de la Mota ${ }^{1}$, M.F. Rivet ${ }^{3}$, F. Sébille ${ }^{1}$ \\ and B. Jouault ${ }^{1}$
}

${ }^{1}$ Laboratoire Subatech, Université de Nantes $/ I N_{2} P_{3} /$ Ecole de Mines de Nantes. F-44072 Nantes Cedex 03. France.

2 Cyclotron Institute, Texas A\&M University, College Station Texas 77843-3366. USA.

${ }^{3}$ Institut de Physique Nucléaire $I N_{2} P_{3}$-CNRS, 91406 Orsay Cedex. France.

\begin{abstract}
${ }^{40} \mathrm{Ar}+{ }^{107} \mathrm{Ag}$ reactions at $27 \mathrm{AMeV}$ and $44 \mathrm{AMeV}$ have been studied within the Landau Vlasov microscopic transport model. In this framework the main characteristics of primary partners of binary collisions are shown to be well described, and information about $\sigma_{n n}$ can be extracted as well as the angular momentum associated with the heavy fragment. Different contributions to the total energy are studied as a function of the reaction time. Our numerical method is compared with the results obtained using experimental techniques like proton spectra. Their impact parameter and energy dependences have been studied and the estimated "temperature parameter" is in agreement with the experimental results.
\end{abstract}




\section{Introduction}

Heavy ion collisions at intermediate energies have been shown to be a powerful tool to investigate the behaviour of out of equilibrium nuclear matter [1]. Indeed, such collisions allow the formation of nuclei under extreme conditions of temperature or/and pressure. It is then possible to drive a system far from its ground state in order to study its stability limits. By analogy with thermodymamics, the maximum temperature that a nucleus can sustain without dissociating could match with the temperature of a phase transition in nuclear matter [2]. To shed light on this problem, different experimental techniques have been developed [3][7].

On one hand, one can investigate the vanishing of low-energy phenomena such as the (in)complete fusion [4]. In this case, the residue characteristics give information about the conditions of their formation. Besides, the source of light particles is well defined and additional insights can be got from emitted light charged particles. In particular, the slope of the single particle spectra can be used as a "thermometer"[5]. On the other hand, binary dissipative collisions have been found an alternative way to produce and study very hot nuclei [8]. This mechanism, which remains less utilized than the previous one, is expected to put more severe constraints on theoretical approaches; indeed kinematical properties of the two main partners of collisions and associated particle emission properties have to be reproduced in a consistent way.

Different theoretical approaches [9]-[18] were focused on the study of the stability properties of nuclear matter. Microscopic transport models [12]-[14] have been shown to give a proper description of the reaction dynamics. In this approximation, a collision term supplements a mean-field evolution equation, in order to include the residual interactions responsible for bringing the system towards equilibrium. These approaches have proved their ability to describe many processes involved in heavy ion collisions, in particular they correctly reproduce most of the reaction mechanisms where heavy fragments are created, such as residue 
cross sections $[15]$ and deep-inelastic reactions $[16,17]$.

In this work we were essentially interested in the study of the interplay between the energies associated to different degrees of freedom, in heavy ion reactions at intermediate beam energies, which are evidenced through the characteristics of fragments and particles detected in an experiment. This investigation was performed in the framework of the Landau-Vlasov model, and recent $\mathrm{Ar}+\mathrm{Ag}$ experimental data around the Fermi energy (27 and $44 \mathrm{AMeV})[19,20]$ were used as a reference.

This paper is organized as follows. In Section 2, we give a survey of the model. In Section 3, fragment and particle characteristics are investigated for the $\mathrm{Ar}+\mathrm{Ag}$ reaction at 27 and $44 \mathrm{AMeV}$. Different contributions to the total energy are analyzed . A "temperature parameter" of the heaviest fragment is calculated, and its impact parameter as well as its energy dependence are studied and compared with the experimental data. Finally, conclusions are given in Section 4.

\section{The model}

The semi-classical Landau-Vlasov model describes the time evolution of the onebody distribution function $f(\vec{r}, \vec{p}, t)$ through the following transport equation:

$$
\frac{\partial f(\vec{r}, \vec{p}, t)}{\partial t}+\{f(\vec{r}, \vec{p}, t), H\}=I_{\text {coll }}(f(\vec{r}, \vec{p}, t))
$$

where $\{$,$\} stands for the Poisson bracket, H$ is the one-body Hamiltonian and $I_{\text {coll }}$ is the Uelhing-Uhlenbeck collision term.

In this approach, nuclear statics and dynamics are treated in a selfconsistent way from a Lagrangian point of view. This means that the one-body distribution function $f(\vec{r}, \vec{p}, t)$ is projected onto a moving basis of coherent states:

$$
f(\vec{r}, \vec{p}, t)=\sum_{i} \omega_{i} g_{\chi}\left(\vec{r}-\vec{r}_{i}\right) g_{\phi}\left(\vec{p}-\vec{p}_{i}\right)
$$


The projection coefficients $\omega_{i}$ are determined by nuclear ground state characteristics. The elements of the basis $g_{\chi}$ and $g_{\phi}$ are gaussians with fixed widths $\chi$ and $\phi$ in, respectively, $\vec{r}$ and $\vec{p}$ spaces. The centers of these gaussians move according to the Ehrenfest equations of motion. This technique, referred to as the full ensemble technique, has been successfully checked by comparisons with analytically solved results [21].

The two-body residual interactions are simulated by making two gaussians collide as if they were hard spheres, provided the collision does not violate the Pauli principle. The gaussian collision cross-section is scaled on the nucleonnucleon cross section so that their averaged mean-free path is the same as for the nucleons. In this work the free isospin and energy dependent nucleon-nucleon cross section is implemented. For more details we refer the reader to Ref. [13].

The Hartree-Fock Hamiltonian has been calculated with the finite range Gogny interaction [22]. This effective force has proven its ability to reproduce static properties of finite nuclei. Without the spin orbit term, its one-body HartreeFock potential in phase space reads:

$$
\begin{gathered}
V_{q}(\vec{r}, \vec{p})=\left[\frac{3}{4}(\gamma+2) \rho^{\gamma+1}(\vec{r})-\frac{3}{4} \gamma \rho^{\gamma-1}(\vec{r})\left(\rho_{n}^{2}(\vec{r})+\rho_{p}^{2}(\vec{r})\right)-\frac{3}{2} \rho^{\gamma}(\vec{r}) \rho_{q}(\vec{r})\right] t_{3} \\
+\sum_{i=1}^{2} \int d \overrightarrow{r^{\prime}}\left[\left(W_{i}+\frac{B_{i}}{2}\right) \rho\left(\overrightarrow{r^{\prime}}\right)-\left(H_{i}+\frac{M_{i}}{2}\right) \rho_{q}\left(\overrightarrow{r^{\prime}}\right)\right] \exp -\frac{\left(\vec{r}-\overrightarrow{r^{\prime}}\right)^{2}}{\eta_{i}} \\
\frac{-2\left(\sqrt{\pi} \eta_{i}\right)^{3}}{(2 \pi)^{3}} \sum_{i=1}^{2} \int d \vec{k}^{\prime} \quad\left[\left(\frac{W_{i}}{2}+B_{i}\right) f_{q}\left(\vec{r}, \vec{k}^{\prime}\right)-\left(\frac{H_{i}}{2}+M_{i}\right) f\left(\vec{r}, \vec{k}^{\prime}\right)\right] \exp -\left[\frac{\left(\vec{k}-\vec{k}^{\prime}\right)^{2} \eta_{i}^{2}}{4}\right]
\end{gathered}
$$

where $\rho_{n}(\vec{r})$ and $\rho_{p}(\vec{r})$ represent, respectively, neutron and proton local densities, $\rho(\vec{r})$ is the total density and $q$ stands for the isospin degree of freedom. In Table 1 the parameters corresponding to the D1-G1 Gogny force are reported. The values of the incompressibility modulus and of the effective mass are, respectively, $K_{\infty}=228 \mathrm{MeV}$ and $m^{\star} / m=0.67$.

In these calculations we use $\mathbf{4 0}$ gaussians per nucleon, which represents a good compromise between reasonable computing time and accuracy. This number of gaussians has been shown to properly describe the macroscopic properties of nu- 
clear matter in infinite nuclear matter calculations [23].

\section{Results}

Recent experimental data [19] have evidenced two main dynamical processes in the $\mathrm{Ar}+\mathrm{Ag}$ reaction at $27 \mathrm{AMeV}$ incident energy. One of them is the incomplete fusion which is the dominant process in central collisions, the other one is binary inelastic scattering which occurs for peripheral and mid-central collisions. Further measurements at $44 \mathrm{AMeV}[20]$ showed that fusion-evaporation and fusion-fission channels disappeared even for the most central events, and that multifragment emission occurs. In addition these experimental results brought strong arguments supporting the idea that, even up to an incident energy of $44 \mathrm{AMeV}$, binary processes are still dominant.

\subsection{Velocity and angular correlations}

In the experimental study of the $\mathrm{Ar}+\mathrm{Ag}$ reactions, coincidences between a heavy fragment, HF (fusion residue or target-like fragment), and a light one LF (deexcitation product or projectile-like fragment) were required. The emission angle of the LF was taken as the reference variable, and for each value of this angle average kinematic quantities related to both fragments were measured [19].The different variables discussed in this subsection are described in Fig 1.

The same reactions were simulated in the framework described in the previous section. A comparison with experimental data could only be performed when at least two fragments were formed in the exit channel, thus excluding the smaller impact parameters eventually leading to fusion. The simulation, which does not include multiparticle correlations, gives estimate of average kinematic properties of the reaction; averaged values of the observables have been experimentally derived. We thus believe that a comparison between calculated and measured values is sound. At higher incident energies it was shown that the flow of nuclear 
matter during a collision was strongly dependent on the in-medium nucleonnucleon cross section, $\sigma_{n n}$. In the same way the rotation angle of the di-nuclear system also reflects a "flow" direction, and should depend on $\sigma_{n n}$. Therefore several values of $\sigma_{n n}$ were implemented in the simulation, in a very simple way: the free $\sigma_{n n}^{f}$, while keeping its momentum and isospin dependences, was scaled by a constant factor which was varied between 0.8 and 1.5 .

An overview of the collisions, for several impact parameters and at two incident energies, is shown in Fig 2. At $27 \mathrm{AMeV}$, fusion occurs for impact parameters below $5 \mathrm{fm}$ [19]. For larger impact parameters a binary dissipative collision is observed; the violence of the collision is linked to the rotation of the system: the projectile-like fragment is deflected to larger angles when b decreases. These deflection angles are negative angles for all impact parameters simulated (see Fig 2). Thus the emission angle of the light fragment, being directly correlated to the impact parameter, will appear as the reference (abscissa axis) in all following figures quoted in this subsection. At $44 \mathrm{AMeV}$, fusion never occurs, even in head-on collisions. For the smaller impact parameters a third fragment (neck emission [24]) appears between the two partners of the binary collision.

In Fig 3 are displayed the emission angle of the heavy fragment in the laboratory $\theta_{H F}$ (top), and its velocity (bottom) as a function of the light fragment angle $\theta_{L F}$ for the $\mathrm{Ar}+\mathrm{Ag}$ reaction at $27 \mathrm{AMeV}$. The thick bars represent the dispersion of the experimental values introduced by the various final $\mathrm{Z}$ of the light fragment $(3 \leq Z \leq 14)$; the lines are the results of the simulation for three values of $\sigma_{n n}$. The dashed lines correspond to $0.8 * \sigma_{n n}^{f}$, the full lines to $\sigma_{n n}^{f}$ and the dotted lines to $1.5 * \sigma_{n n}^{f}$. The experimental correlations are well reproduced by the simulation, irrespective of the value of $\sigma_{n n}$. In fact, the calculated observables $\left(V_{H F}, \theta_{H F}\right.$ and $\left.\theta_{L F}\right)$ are all strongly related with the relative weight between the attractive action of the nuclear force and the repulsion due to residual interactions. Our calculation shows that a strong experimental selectivity in impact parameter would be needed to constrain $\sigma_{n n}$.

On the left part of Fig 4, the relative velocities between the two fragments 
are displayed, at 27 and $44 \mathrm{AMeV}$ as a function of $\theta_{L F}$. Conversely to the results depicted in Fig 3, the experimental relative velocities are independent of the final $\mathrm{Z}$ of the light fragment. When expressed as $\left(V_{\text {rel }}-V_{c}\right) /\left(V_{p}-V_{c}\right)$, where $V_{p}$ and $V_{c}$ stand for the projectile and Coulomb velocities, a value close to 1 refers to a quasi-elastic reaction, whereas a value near 0 is characteristic of a completely damped collision. As expected for binary dissipative collisions, the more violent is the collision, the more damped is the relative motion. The different lines show the results of the simulations: this variable $\left(V_{\text {rel }}\right)$ exhibits a clear sensitivity to $\sigma_{n n}$, an increase of the collision rate increasing the damping. At $27 \mathrm{AMeV}$, the best agreement with experimental data is obtained for $\sigma_{n n}=1.5 * \sigma_{n n}^{f}$, while at $44 \mathrm{AMeV}$ the calculated curve closest to the experimental values correspond to $\sigma_{n n}=0.8-1.0 \sigma_{n n}^{f}$. At this energy, the comparison is restricted to $\theta_{L F} \leq 20^{\circ}$ for two reasons: i) in the calculation we observe the appearance of neck emission for impact parameters smaller than $5 \mathrm{fm}$ which reduces the angular range explored to 20 degrees. ii) Experimentally the binary character of the collisions becomes doubtful for $\theta_{L F}>20^{\circ}$ ). These results are a first experimental hint of a variation of $\sigma_{n n}$ in the nuclear matter with the incident energy, as suggested in ref. $[25,26]$.

The right part of Fig 4 presents the recoil velocity of the binary system before it breaks in two fragments, normalized to the c.m. velocity of the initial system. This variable, for an asymmetric system in direct kinematics, allows to quantify the amount of preequilibrium emission: $V_{r e c} / V_{c m}$ is equal to 1 if there is no preequilibrium emission, and becomes smaller if there is a dominant preequilibrium emission of projectile nucleons. At both energies the experimental values show a growing importance of preequilibrium emission with the increasing violence of the collision. This trend is well reproduced by the simulations, with no dependence on $\sigma_{n n}$ at $27 \mathrm{AMeV}$. At $44 \mathrm{AMeV}$, the calculation which best agrees with the experimental data is again that with $\sigma_{n n}=0.8-1.0 \sigma_{n n}^{f}$, but the amount of preequilibrium emission is slightly overestimated. Very low values $\left(V_{\text {rec }} / V_{c m} \sim 0.6\right)$ are reached in the experiment, which may be due, as just mentioned above, to the presence of a (non measured) third fragment; the simulation, although for 
small angles, reproduces these small values when only the fastest of the two light fragments produced in the exit channel is considered.

To conclude this section, it can be stated that the LV simulation implemented with the Gogny force well predicts the dynamics of the $\mathrm{Ar}+\mathrm{Ag}$ reactions at $27 \mathrm{AMeV}$, over the whole impact parameter range, provided that the nucleon-nucleon cross section is enhanced by $50 \%$. This enhancement disappears when the incident energy is raised to $44 \mathrm{AMeV}$. This trend goes along with nucleon-nucleon cross-section calculations in nuclear medium with the Brueckner G-matrix $[25,26]$, which predict an enhancement with respect to $\sigma_{n n}^{f}$, for low bombarding energies.

\subsection{Angular momentum}

In Fig. 5 the angular momentum $\mathrm{J}$ of the primary heavy fragment just after reseparation is displayed as a function of the impact parameter. This observable was calculated with different scalings of the nucleon-nucleon cross-section for the Ar+Ag system at the same incident energies as before: 27 (right) and 44 (left) $\mathrm{AMeV}$. For both energies the full line corresponds to $\sigma_{n n}^{f}$, dashed line to $0.8 \sigma_{n n}^{f}$ and dotted line to $1.5 \sigma_{n n}^{f}$. A first remark is that, for a given incident energy and impact parameter, there is no clear influence of $\sigma_{n n}$ on $\mathrm{J}$, the small variations observed in Fig 5 may only reflect fluctuations in the calculated values. More interesting is the evolution of $\mathrm{J}$ with the impact parameter and the incident energy. At $27 \mathrm{AMeV}, \mathrm{J}$ increases with $\mathrm{b}$ as long as incomplete fusion occurs and then decreases when binary inelastic collisions take place. A maximum value around $60 \hbar$ is calculated. A $44 \mathrm{AMeV}$ where no fusion occurs, J increases up to $b \sim 3 f m$, keeps a rather constant value $(\sim 30-35 \hbar)$ up to $\mathrm{b}=8 \mathrm{fm}$ before decreasing for the most peripheral collisions. When comparing the results for both incident energies, one notices that for a given impact parameter $\mathrm{J}$ is lower when the incident energy is higher. In summary, maximum calculated angular momentum transfers are observed for mid-central collisions at moderate incident energies. Conversely in peripheral collisions at high incident energies, the angular 
momentum transferred to the heavy fragment is very small.

These calculated trends are in qualitative agreement with the results of $[19$, 20]. Indeed, a strong anisotropy was observed for $\alpha$-particle emission from the HF at $27 \mathrm{AMeV}$; the values of $\mathrm{J}$ which can be derived from the observables quoted in tables 2,3,4,6 of ref [19] are around 55-65 $\hbar$, and are plotted in Fig 5 with crosses, for impact parameters deduced from the HF velocity (see next section). Except for $b=8 \mathrm{fm}$, a fair agreement between calculations and experiment is observed. Conversely, at $44 \mathrm{AMeV}$ the measured $\alpha$-particle angular distributions are compatible with an isotropic emission [20], indicating a much lower angular momentum of the emitting HF, in agreement with the calculated trend.

\subsection{Characteristics of single particle spectra}

The studied $\mathrm{Ar}+\mathrm{Ag}$ reaction $[19,20]$ gives us the opportunity to compare theoretical calculations with some semi-inclusive data (coincidences between the heavy fragment and the light particles it emits). Indeed, at each energy, events have been classified as a function of the violence of the reaction through the velocity of the heavy fragment. We have imposed the same constraints on our calculated results and each class of events has been linked with a single impact parameter. At 27(44)AMeV, these are $b=5(3) \mathrm{fm}, b=7(5) \mathrm{fm}$ and $b=8(8) \mathrm{fm}$. In Fig 6 calculated single proton spectra are shown for the $\mathrm{Ar}+\mathrm{Ag}$ reaction at $27 \mathrm{AMeV}$, for different impact parameters. To this end, the double differential cross-section $d^{2} N / d \Omega d E$ has been calculated by assuming that the source velocity is along the beam axis, in the same way as in the experiment. The spectra were calculated at 90 degrees in the center of mass where the most important statistics is available.

A "temperature parameter" at the time at which the heavy fragment breaks up is extracted from a fitting of the differential-cross section with the Weisskopf function, which is given by the following expression:

$$
\frac{d^{2} \sigma}{2 \pi \sin (\theta) d E d \theta}=a_{1} \frac{E-B}{T_{a p p}{ }^{2}} \exp -\left(\frac{E-B}{T_{a p p}}\right)
$$

Here, $a_{1}$ is a scaling coefficient, $B$ stands for the Coulomb barrier and $T_{a p p}$ for the 
apparent temperature parameter. We report in Table 2, our calculated values of $T_{a p p}$ for the heavy fragment and the corresponding quotient $V_{H F} / V_{C M}$, for different impact parameters, at each incident energy. The estimated temperatures are fairly independent of the centrality of the collision and of the incident energy. These results agree with the experimental yields [20] and support the underlying idea of a possible saturation of the heavy fragment temperature around $7 \mathrm{MeV}$.

This finding can be interpreted through the analysis of the different contributions to the total energy of the colliding system. The kinetic energy can be calculated as:

$$
E_{k i n}=E_{c o l l}+E_{i n t}+E_{0}
$$

where $E_{\text {coll }}$ is the collective energy, $E_{\text {int }}$ is the energy associated to the intrinsic degrees of freedom at finite temperature, and $E_{0}$ is the intrinsic energy at zero temperature, calculated according to Ref.[18].

In Fig.7.a) the time evolution of these different contributions to the total kinetic energy is shown for the $\mathrm{Ar}+\mathrm{Ag}$ collision at $44 \mathrm{Mev} / \mathrm{n}$ and $\mathrm{b}=3 \mathrm{fm}$. For sake of simplicity $E_{0}$ is not displayed. The dashed line represents the intrinsic energy of heavy fragments, the solid line is the total intrinsic energy including free-particles, and the dotted-line is the sum of the preceding quantity plus the collective energy. The time at which the system becomes locally equilibrated $[18], t_{e q}$, and the interval of time for the separation of primary partners $t_{\text {sep }}$ are explicitly indicated. We have to point out here that the time $t_{e q}$ differs from the global equilibration time $(120 \mathrm{fm} / \mathrm{c})$ typically used to connect experimental data to calculations [19]. The energy of particles which were emitted before $t_{e q}$ is represented by a vertical bar and was called $E_{e q}$. In this picture, the energy exchanges between the different degrees of freedom are put in evidence. Indeed, the kinetic energy is initially stored in the collective modes and it is progressively transferred to the intrinsic ones. For asymptotic times, the resulting heavy fragments have decayed and the total excitation energy has been carried away by the emitted particles. The heavy fragment excitation energy oscillates between two maxima located around the equilibration and the separation times, 
reflecting energy exchanges between thermal and compressional modes. This fact can also be observed in Fig.7.b) where the time evolution of the mean local density $\rho$ of the heavy cluster, normalized to the saturation value $\rho_{0}$, is represented for the same reaction. In this picture, the heavy fragment passes through two compressed stages, one at about $t_{e q}$ and the other around $t_{\text {sep }}$. At this point, the independence of the slope of the particle spectra with respect to the incident energy can be related to the energy carried out by those emitted particles which are not pre-equilibrium particles, at $t=t_{\text {sep }}$ (see Fig.7.a)). This quantity, referred to as $E_{e}$, is the only contribution which is independent of the incident energy for a given impact parameter. This aspect can be observed in Table 3, where we have reported the number of emitted particles $n\left(t_{e q}\right)\left(n\left(t_{\text {sep }}\right)\right)$ at the equilibration (separation) time, their difference $\Delta n=n\left(t_{\text {sep }}\right)-n\left(t_{e q}\right)$, and $E_{e}=E\left(t_{\text {sep }}\right)-E\left(t_{e q}\right)$, for different impact parameters and at the two concerned incident energies.

In this way, even though for all $\mathrm{b}$ at $27 \mathrm{AMeV}$ the energy of emitted particles at $t_{\text {sep }}$ is lower than at $44 \mathrm{AMeV}$, the preequilibrium component is also lower, and the separation time larger, in such a way that the resulting $E_{e}$ is of the same order in both cases. On the other side, from Table 3 we observe that the quotient $E_{e} / \Delta n$ is nearly constant, indicating that the average energy per nucleon carried away by the emitted particles at $t>t_{e q}$ is nearly independent of $\mathrm{b}$ and $\mathrm{E}$.

In Fig. 8 we have represented the time evolution of the emitted particle spectra at 90 degrees, at $44 \mathrm{AMeV}$ incident energy and $\mathrm{b}=3 \mathrm{fm}$. From this picture we can observe that the slope for asymptotic times is already built up at the separation time $t_{\text {sep }}=140 \mathrm{fm} / \mathrm{c}$, and that the subsequent particle emission has no effect on the evolution of the slope. A consequence of this is the difficulty to track down a direct connection between the evaporated particles and the amount of energy which has been deposited in the system during the reaction. Assuming that this energy had to be "thermalized" in the heavy fragment ( $E_{d}$ in Fig.7.a), then the slope of spectra, created before the separation time, does not provide a good estimation of this deposited energy. 


\section{Conclusions}

In this work we have performed an analysis of the thermalization processes in a heavy-ion collision at intermediate energies, motivated by the recent interest of many experiments in characterizing the stability limits of excited nuclei. We have in particular considered the reaction $\mathrm{Ar}+\mathrm{Ag}$ at 27 and $44 \mathrm{AMeV}$ beam energies, performed at GANIL, from which a considerable amount of data is presently available [20]. We have simulated the nuclear dynamics within the framework of the semi-classical Landau-Vlasov model, and the relevant observables were numerically calculated following the experimental constraints. In a first time, the analysis of angular and velocity correlations between the two partners of binary collisions has been shown to be a good test of coherence with respect to the experiment. In this picture, the relative velocity between the two partners is a measure of the degree of dissipation in the collision, and was shown to be sensitive to $\sigma_{n n}$. Our calculations follow correctly the experimental trends for a $\sigma_{n n}$ value $50 \%$ higher than the free one for $27 \mathrm{AMeV}$ beam energy. However more precise experimental impact parameter dependence of the observables discussed in this paper have to be obtained to strengthen this enhancement. Such accurate measurements will be available using new generation $4 \pi$ detectors [27].

The study of the angular momentum of the primary HF puts in evidence the existence of dynamical mechanisms ruling angular momentum transfers, in such a way that an enhancement of $J$, and then more anisotropy, can be expected at lower energies. The experimental results are in agreement with the calculated trends.

The analysis of single particle spectra in terms of impact parameter for the two energies sticks closely to the experimental trends. The slope of the spectra, or "temperature parameter", appeared to be rather independent of the incident energy and of the impact parameter. This result is interpreted here in terms of the interplay between the energies associated to different degrees of freedom. Indeed, the previous analysis pointed out that the slopes of the spectra were constructed 
very early in the reaction, before the system has broken into fragments, and that the energy carried away by the emitted particles (apart from pre-equilibrium particles) was independent of the incident energy, and scaled with the number of these particles at each impact parameter.

\section{Acknowledgements:}

One of us (FH) wants to thank the Robert A. Welch Foundation and the United State Department Of Energy (grant $N^{\circ}$ DE-FG05-86ER40256) for their support.

\section{References}

[1] V. De La Mota, F. Sébille, M. Farine, B. Remaud and P. Schuck, Phys. Rev. C46 (1992) 677.

[2] S. Levit and P. Bonche, Nucl. Phys. A437 (1985) 2.

[3] J. Galin et al, Proc. of the Symp. on hot Nuclei, Dallas, World Scientific (1988) 241.

[4] B. Borderie and M.F. Rivet, Z. Phys. A321 (1985) 703; C. Volant, M. Conjaud, S. Harar, M. Mostefai, E.C. Pollaco, Y. Cassagnou, R. Dayras, R. Legrain, G. Klotz-engmann and H. Oeschler, Phys. Lett. B195 (1987) 22.

[5] M. Gonin et al, Phys. Rev. C42 (1990) 2125.

[6] K. Hagel et al, Phys. Rev. Lett. 68 (1992) 2141.

[7] G.F. Peaslee et al, internal report MSUCL-918 (1994).

[8] B. Borderie, M.F. Rivet and L. Tassan-Got, Ann. Phys. Fr. 15 (1990) 287 and references therein. 
[9] E. Suraud, C. Grégoire and B. Tamain; Prog. Part. Nucl. Phys. 23 (1989) 357.

[10] B. Remaud, C. Grégoire, F. Sébille, and P. Schuck; Nucl. Phys. A488 (1988) 423.

[11] H.M. Xu, P. Danielewicz and W.G. Lynch, Phys. Lett. B299 (1993) 1993.

[12] G. Bertsch and S. Das Gupta, Phys. Rep. 160 (1988) 190; W. Cassing, K. Niita and S.J. Wang, Z. Phys. A341 (1988) 439; J. Aichelin, Phys. Rev. C33 (1986) 537.

[13] C. Grégoire, B. Remaud, F. Sébille, L. Vinet and Y. Raffray; Nucl. Phys. A465 (1987) 317.

[14] J. Aichelin, Phys. Rep. 202 (1991) 233.

[15] H.M. Xu, W.G. Lynch, P. Danielewicz and G.F. Bertsch, Phys. Rev. C65 (1990) 843.

[16] M.F. Rivet, B. Borderie, C. Grégoire, D. Jouan, and B. Remaud; Phys. Lett. B 215 (1988) 295.

[17] F. Haddad, G. Royer, F. Sébille and B. Remaud, Nucl. Phys. A571 (1994) 459.

[18] P. Abgrall, F. Haddad, V. De La Mota and F. Sébille, Phys. Rev. C49 (1994) 1040.

[19] D. Jouan, B. Borderie, M.F. Rivet, C. Cabot, H. Fuchs, H. Gauvin, C. Grégoire, F. Hanappe, D. Gardes, M. Montoya, B. Remaud, F. Sébille, Z. Phys. A340 (1991) 63.

[20] M.F. Rivet et al, Proceedings of the XXXI Int. Winter Meeting on Nuclear Physics, Bormio (1993), (Riserca Scientifica ed Educazione Permanente, Milano, 1993) p. 92. 
[21] G. Welbke, R. Malfliet, C. Grégoire, M. Prakash and E. Suraud; Phys. Rev. C40 (1989) 2611.

[22] J. Déchargé and D. Gogny,Phys. Rev C21 (1980) 1568.

[23] D. Idier, M. Farine, B. Remaud and F. Sébille; Ann. Phys. Fr. 19 (1994) 159.

[24] J. Töke, B. Lott, S.P. Baldwin, B.M. Quednau, W.U. Schröder, L.G. Sobotka, J. Barreto, R.J. Charity, L. Gallamore, D.G. Sarantites, D.W. Stracener and R.T. De Souza, Nucl. Phys. A583 (1995) 519; L. Stuttgé, J.C. Adloff, B. Bilwes, R. Bilwes, F. Cosmo, M. Glaser, G. Rudolf, F. Scheibling, R. Bougault, J. Colin, F. Delaunay, A. Genoux-Lubain, D. Horn, C. Le Brun, J.F. Lecolley, M. Louvel, J.C. Steckmeyer and J.L. Ferrero, Nucl. Phys. A539 (1992) 511.

[25] A. Bohnet, N. Ohtsuka, J. Aichelin, R Linden and A.Faessler, Nucl. Phys. A494 (1989) 349.

[26] J. Cugnon, A. Lejeune and P. Grangé, Phys. Rev. C35 (1987) 861.

[27] J.Pouthas et al, NIM A 357 (1995) 418. 


\section{Figure Captions}

Fig.1 Schematic view of the different velocities defined in our analysis.

Fig.2 Mean density in the reaction plane for the $\mathrm{Ar}+\mathrm{Ag}$ reaction at different impact parameters at (a) $27 \mathrm{AMeV}$ and (b) $44 \mathrm{AMeV}$ incident energies for $\sigma_{n n}^{f}$.

Fig.3 Heavy residue deflection angle (top) and velocity (bottom), in the center of mass frame, as a function of the LF angle in $\mathrm{Ar}+\mathrm{Ag}$ at $27 \mathrm{AMeV}$. Experimental data are represented by large bars [19].

Fig.4 Recoil velocity of the system normalized to the center of mass velocity (right) and relative velocity of the two partners (see text) (left), at $27 \mathrm{AMeV}$ (top) and $44 \mathrm{AMeV}$ (bottom) for the $\mathrm{Ar}+\mathrm{Ag}$ reaction. Crosses represent experimental data $[19,20]$.

Fig.5 Mean angular momentum as a function of the impact parameter for two energies in the $\mathrm{Ar}+\mathrm{Ag}$ reaction. Crosses represent experimental data [19].

Fig.6 Single proton spectra in $\mathrm{Ar}+\mathrm{Ag}$ at $27 \mathrm{AMeV}$ for different impact parameters.

Fig.7 (a) Time evolution of kinetic energy contributions in $\mathrm{Ar}+\mathrm{Ag}$ at $44 \mathrm{AMeV}$, $b=3 \mathrm{fm}$ : heavy fragment excitation energy (dashed line), total excitation energy (solid line), total kinetic energy (dotted line). (b) Time evolution of the normalized mean density for the same reaction.

Fig. 8 Time evolution of particle spectra at $90 \mathrm{deg}$. in the center of mass frame for $\mathrm{Ar}+\mathrm{Ag}$ reaction at $44 \mathrm{AMeV}$ and $\mathrm{b}=3 \mathrm{fm}$. 
Figure 1

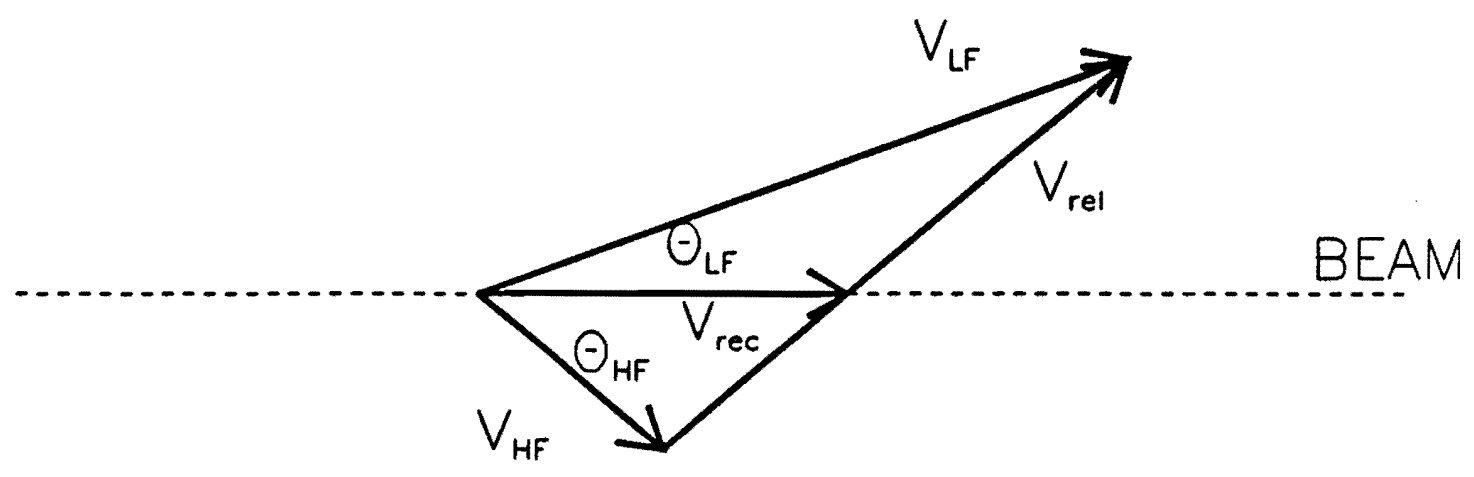


Figure 2-a

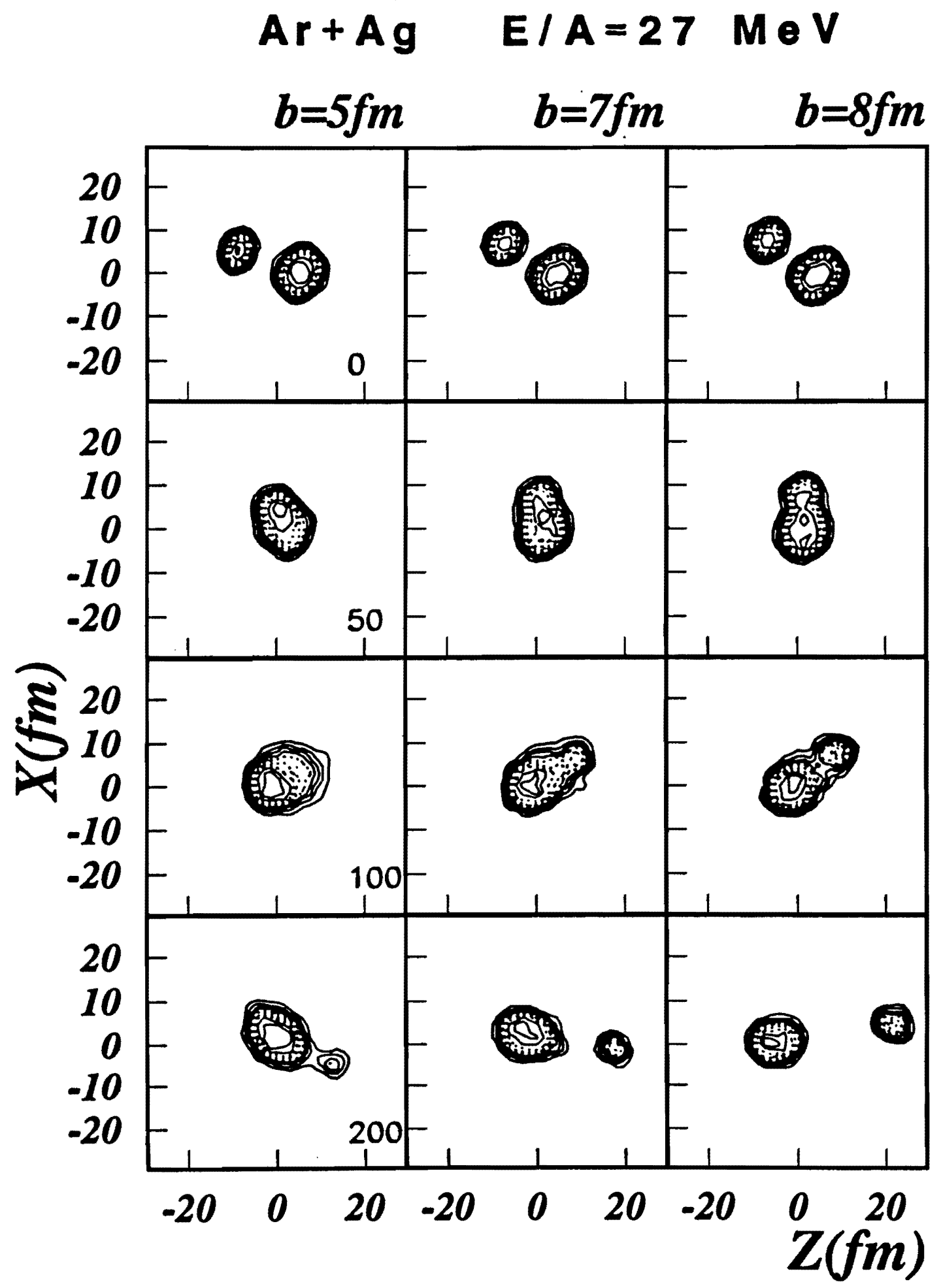


Figure 2-b

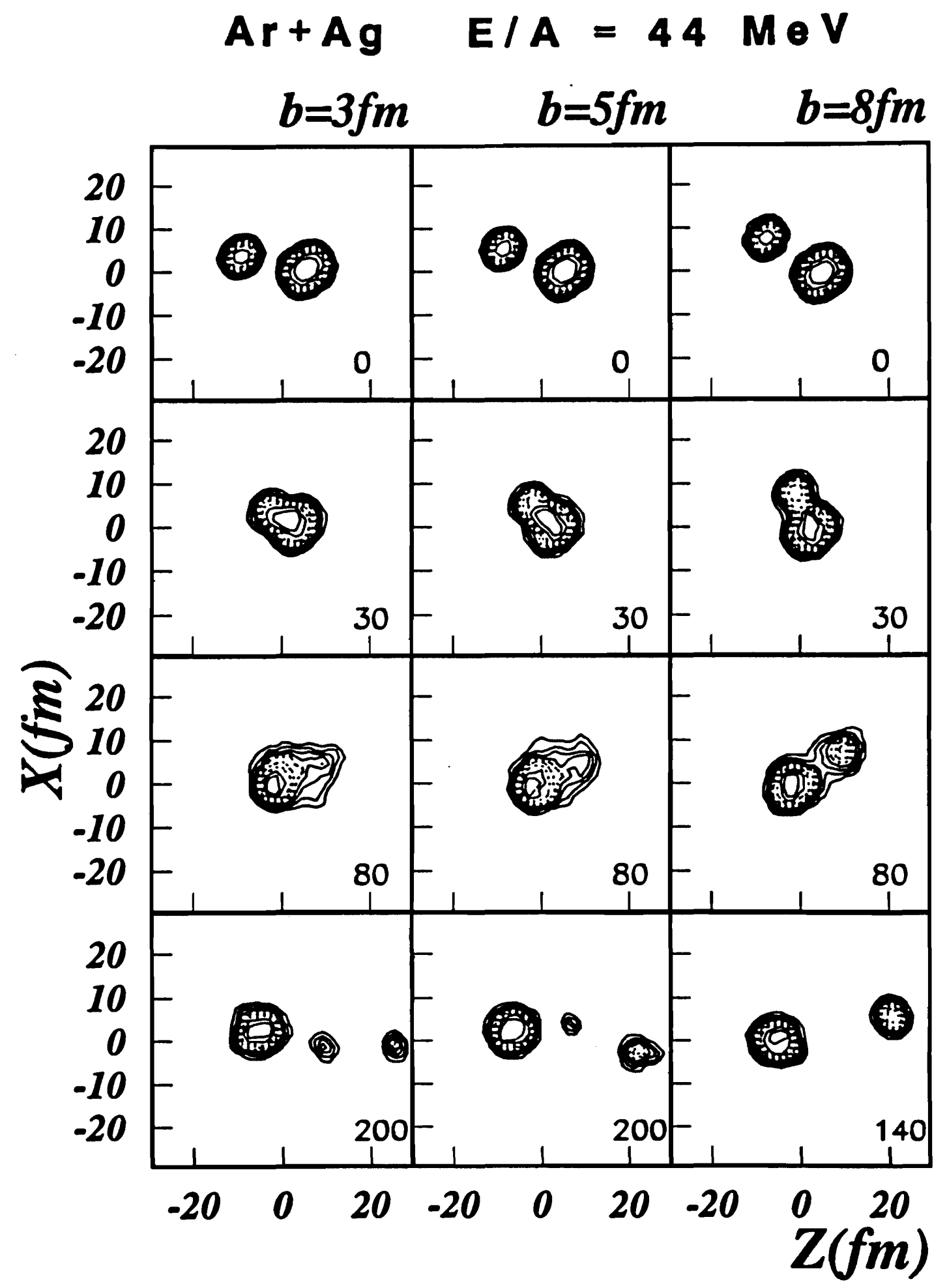


Figure 3

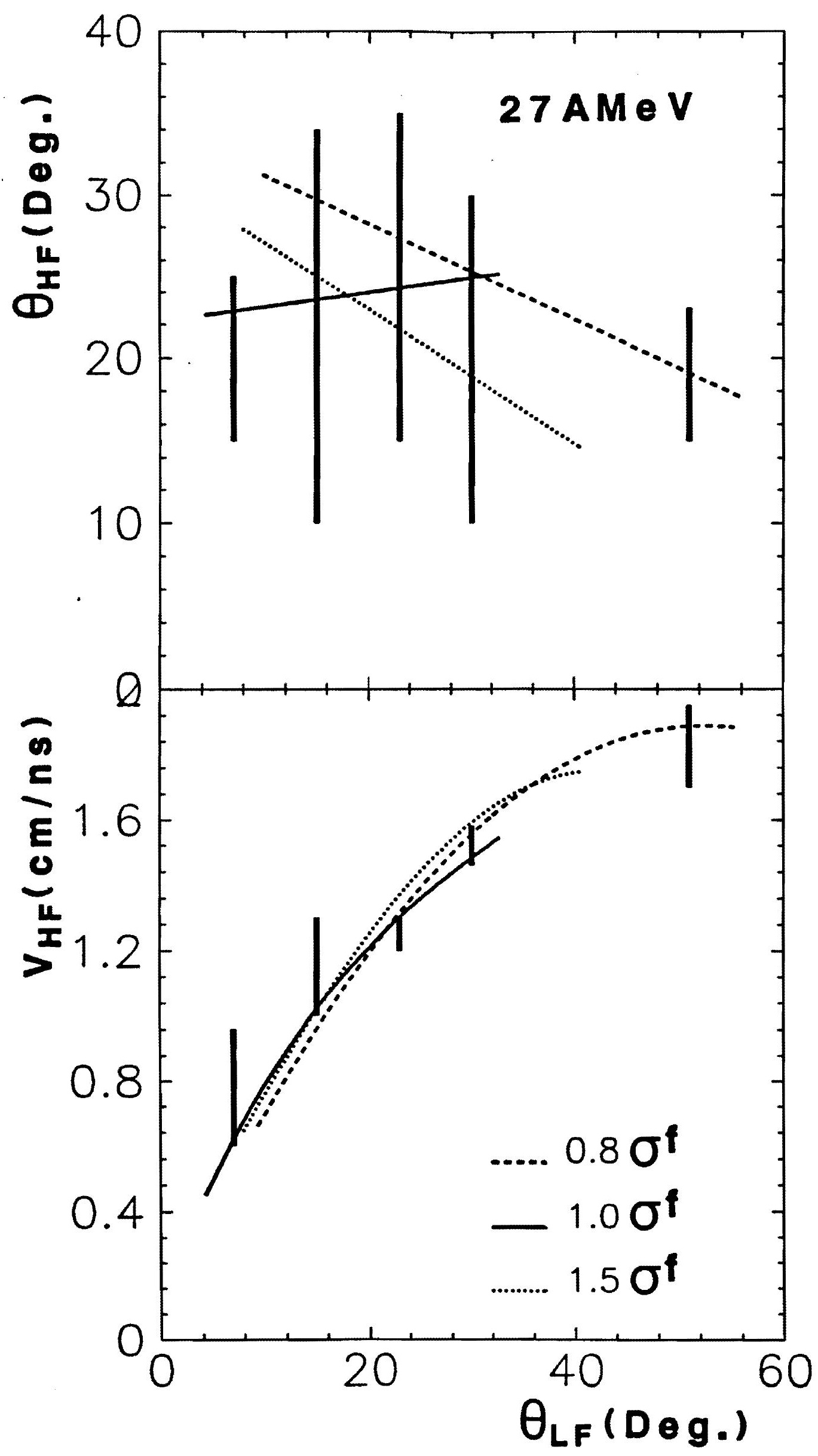


Figure 4-a
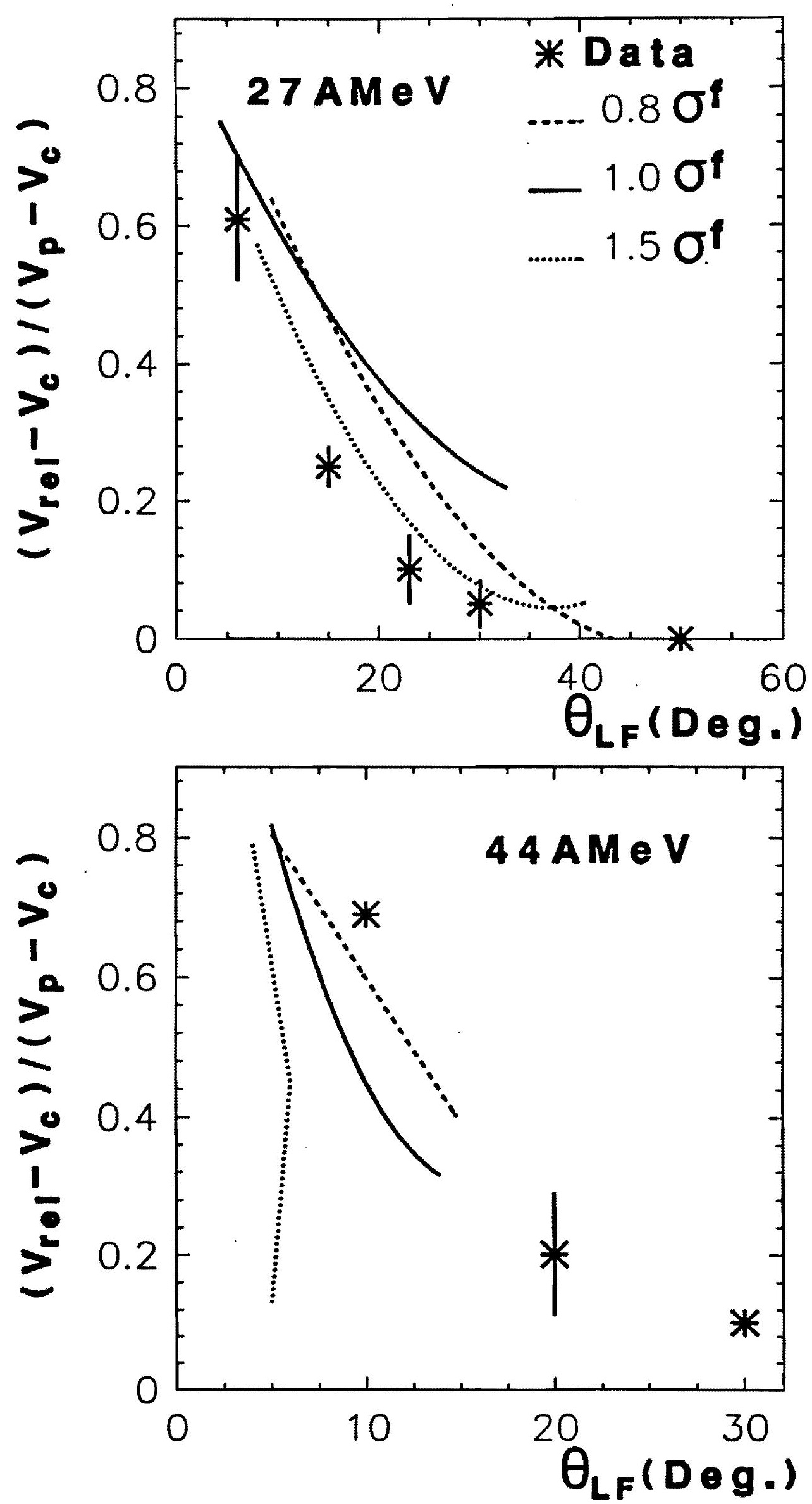
Figure 4-b
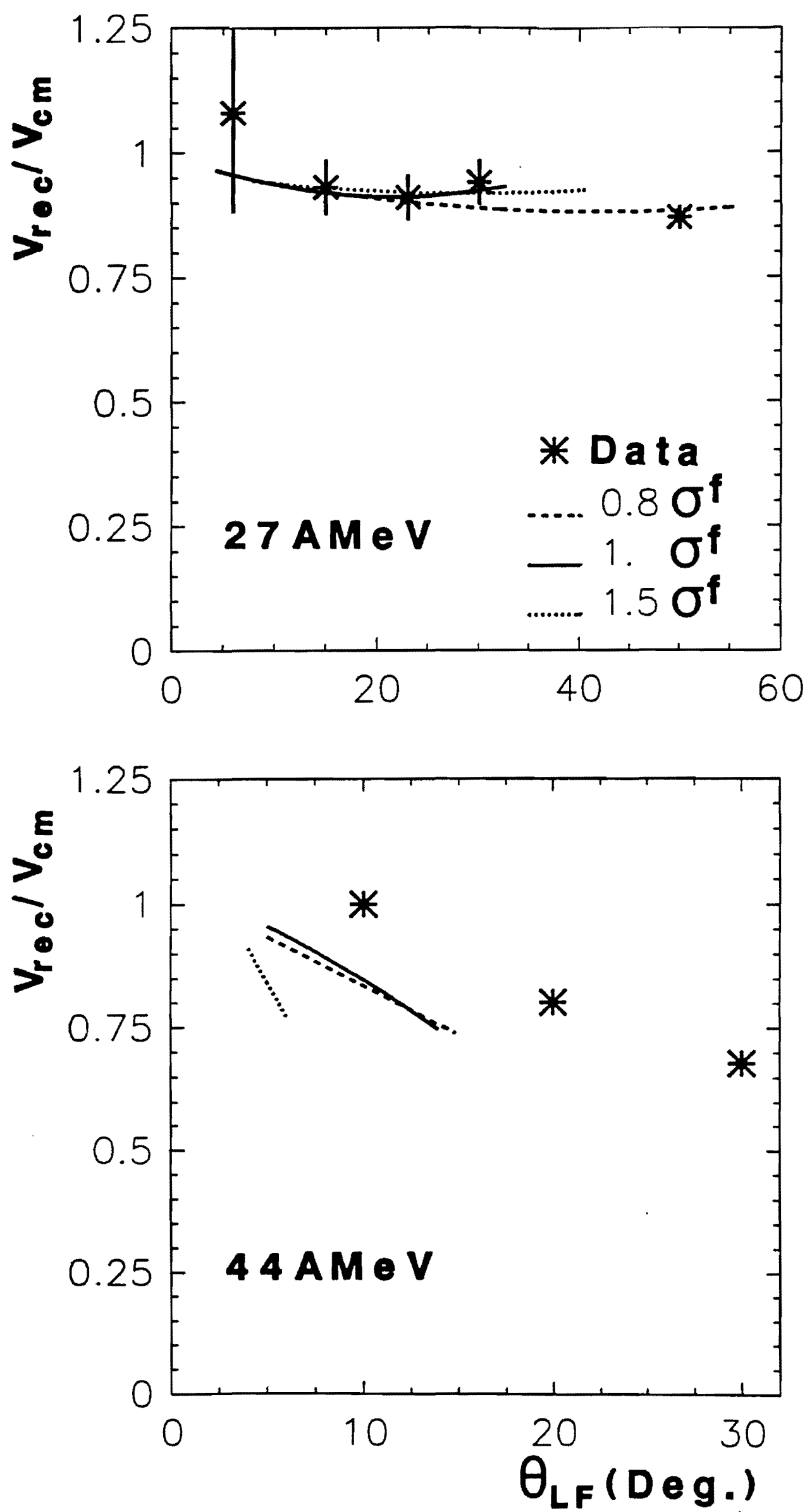
Figure 5

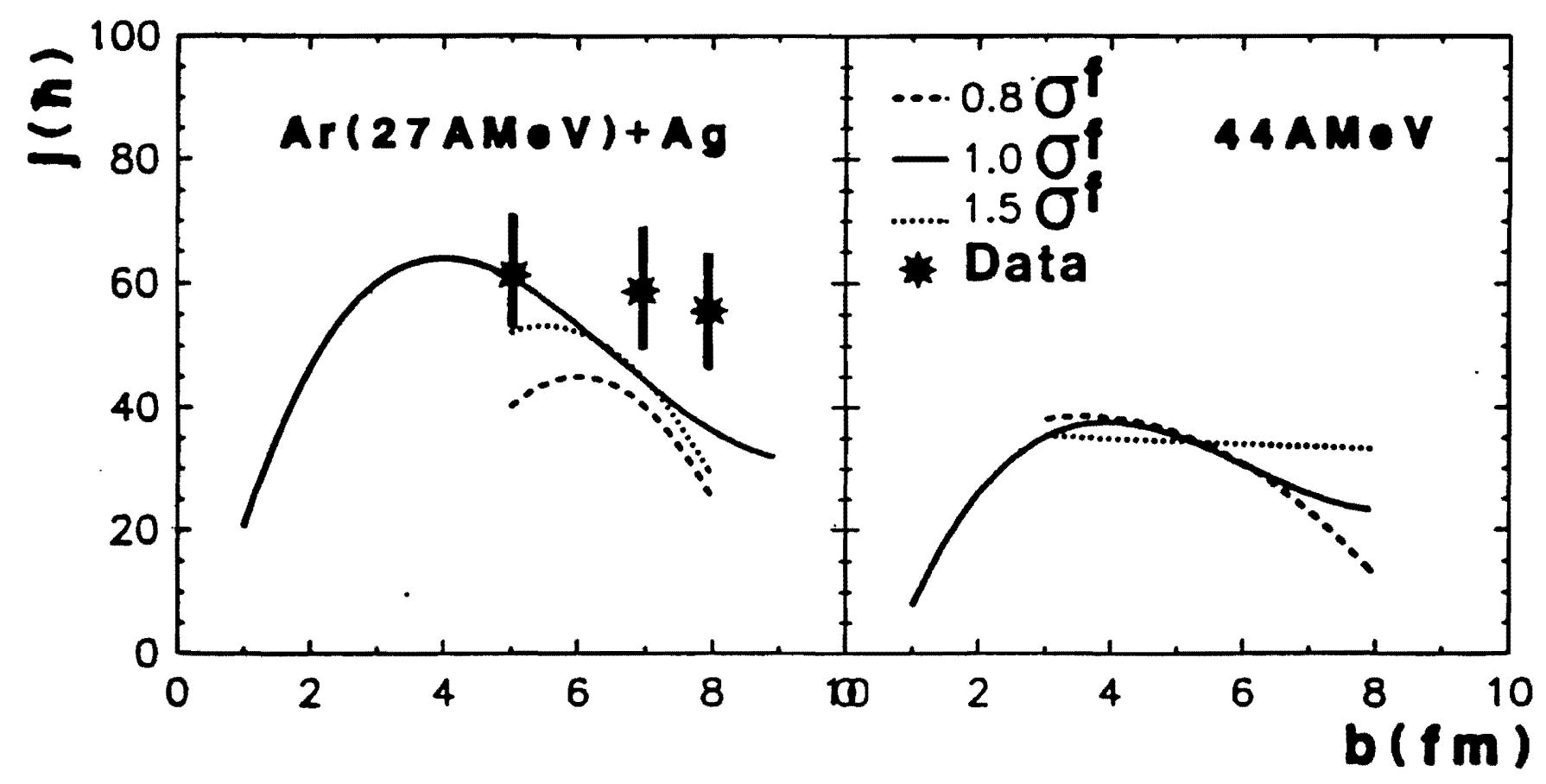


Figure 6

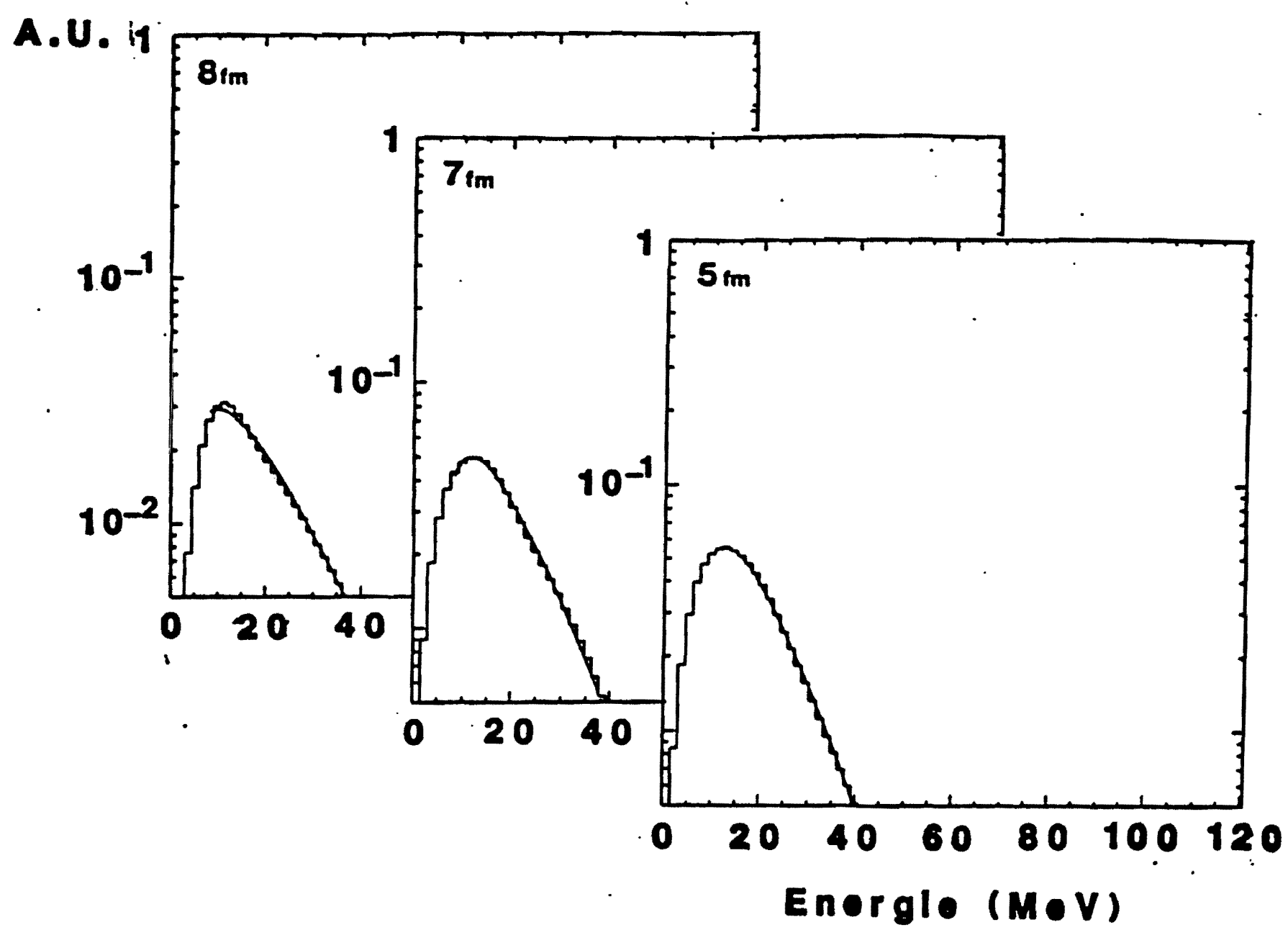


Figure 7-a

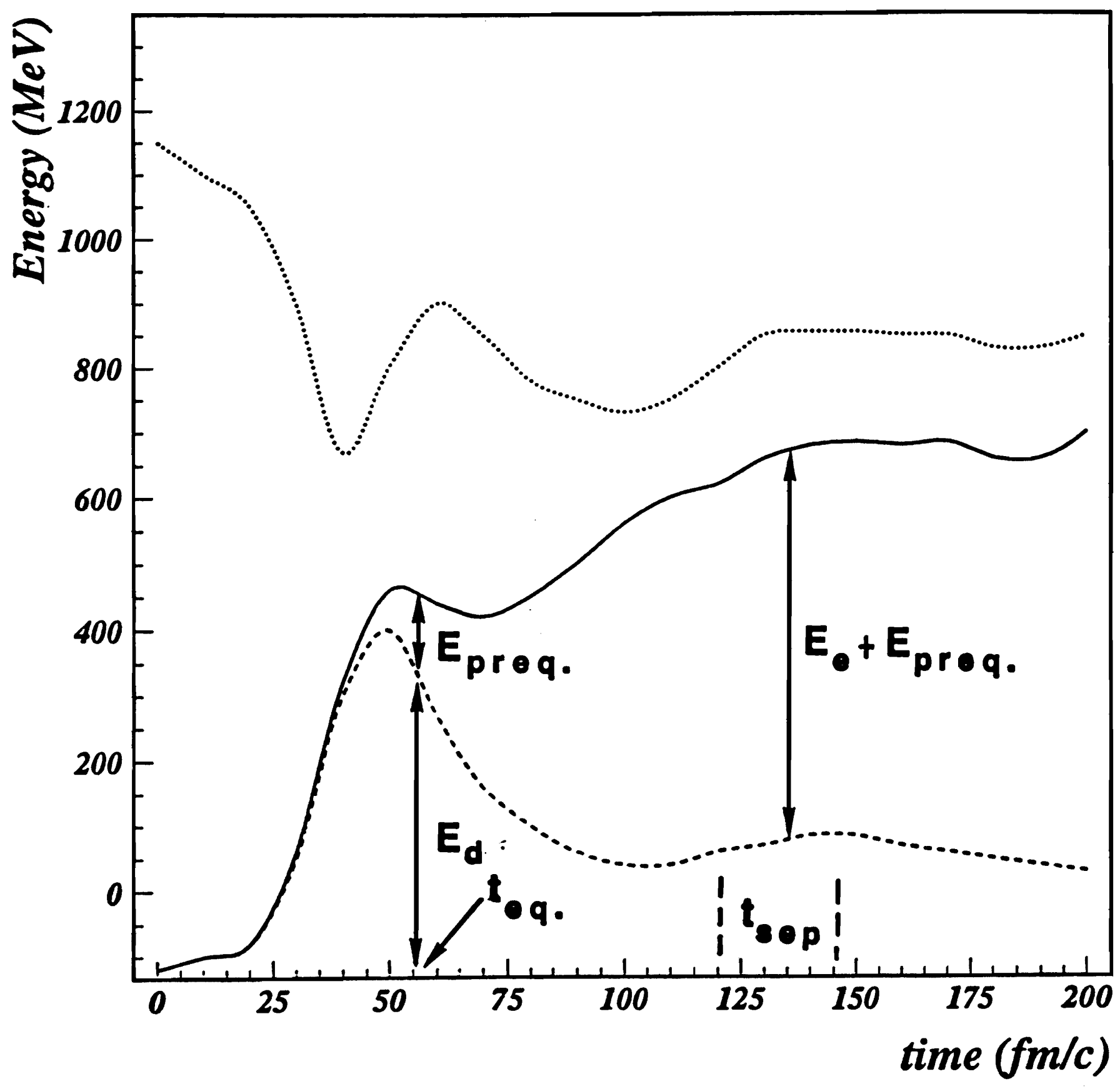


Figure 7-b

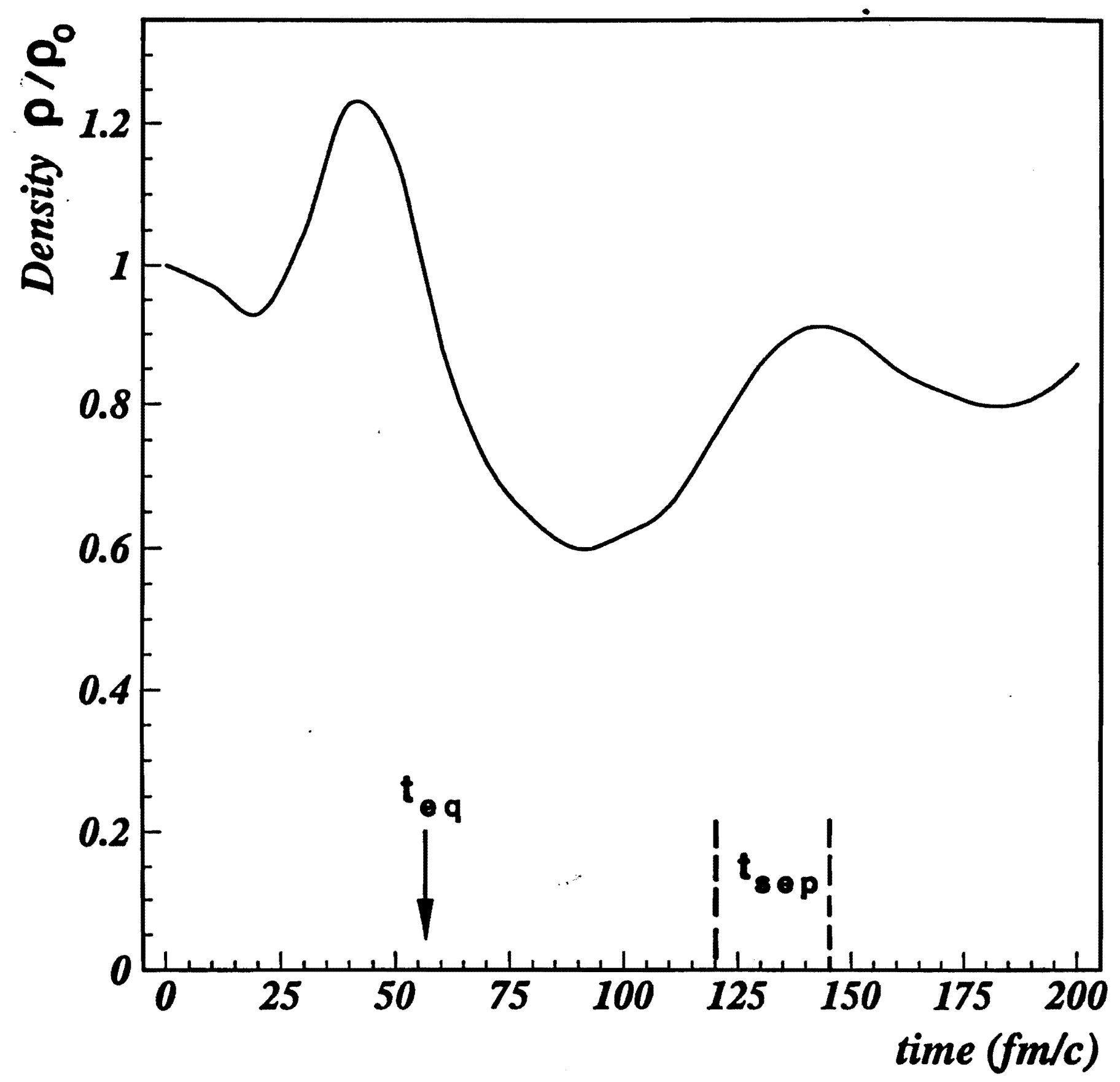


Figure 8

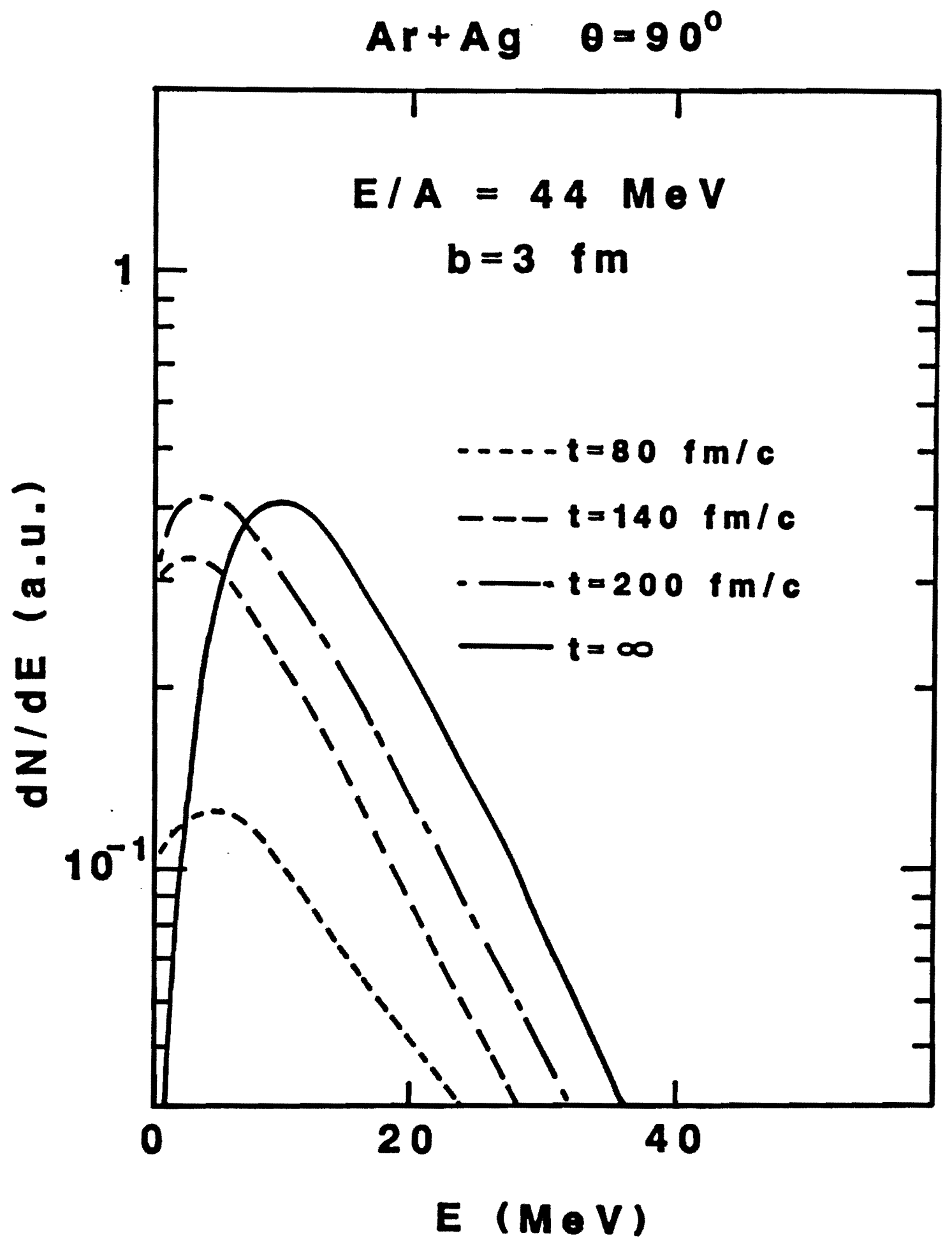




\section{Table Captions}

Table 1: Parameters of the Gogny D1-G1 interaction.

Table 2: Calculated values of $T_{a p p}$ for the heavy fragment at different $V_{H F} / V_{C M}$ values and incident energies.

Table 3: Number of emitted particles at equilibration and separation times respectively $n\left(t_{e q}\right)$ and $n\left(t_{\text {sep }}\right)$, their difference $\Delta n=n\left(t_{\text {sep }}\right)-n\left(t_{e q}\right)$, and $E_{e}=$ $E\left(t_{\text {sep }}\right)-E\left(t_{e q}\right)$ at 27 and $44 \mathrm{AMeV}$. 


\begin{tabular}{ccccccccc}
\hline Table 1 & $i$ & $\eta_{i}$ & $W_{i}$ & $B_{i}$ & $H_{i}$ & $M_{i}$ & $T_{3}$ & $\gamma$ \\
\hline D1-G1 & 1 & 0.7 & -402.4 & -100. & -496.2 & -23.56 & 1350. & $\frac{1}{3}$ \\
& 2 & 1.2 & -21.3 & -11.77 & 37.27 & -68.81 & 1350. & $\frac{1}{3}$ \\
\hline
\end{tabular}

Table 1: 


\begin{tabular}{|c|c|c|c|c|c|}
\hline $\mathbf{b}(\mathbf{f m})$ & $\mathbf{3}$ & $\mathbf{5}$ & $\mathbf{7}$ & $\mathbf{8}$ & $\mathbf{E} / \mathbf{A}(\mathbf{M e V} / \mathbf{n})$ \\
\hline $\mathrm{V}^{\mathrm{hr}} / \mathrm{V}^{\mathrm{cm}}$ & & 0.94 & 0.54 & 032 & 27 \\
& 0.61 & 0.51 & & 0.20 & 44 \\
\hline $\mathrm{T}_{\text {app }}(\mathrm{MeV})$ & & 6.8 & 6.8 & 7.2 & 27 \\
& 6.6 & 6.5 & & 7.2 & 44 \\
\hline
\end{tabular}

Table 2: 


\begin{tabular}{|l|l|c|c|c|c|}
\hline E/A & & $\mathbf{3 f m}$ & $\mathbf{5 f m}$ & $\mathbf{7 f m}$ & $\mathbf{8 f m}$ \\
\hline \multirow{4}{*}{$27 \mathrm{MeV}$} & $\mathbf{n}$ (teq) & & 2 & 2 & 1 \\
\cline { 2 - 6 } & $\mathbf{n}$ (tsep) & & 22 & 14 & 11 \\
\cline { 2 - 6 } & $\Delta \mathbf{n}$ & & 20 & 12 & 10 \\
\cline { 2 - 6 } & $\mathbf{E}$ (tsep)-E(teq) & & $\sim 400$ & $\sim 250$ & $\sim 200$ \\
\hline \multirow{4}{*}{$44 \mathrm{MeV}$} & $\mathbf{n}$ (teq) & 13 & 8 & & 4 \\
\cline { 2 - 6 } & $\mathbf{n}$ (tsep) & 35 & 29 & & 14 \\
\cline { 2 - 6 } & $\Delta \mathbf{n}$ & 22 & 21 & & 10 \\
\cline { 2 - 7 } & $\mathbf{E}$ (tsep)-E(teq) & $\sim 400$ & $\sim 400$ & & $\sim 200$ \\
\hline
\end{tabular}

Table 3: 